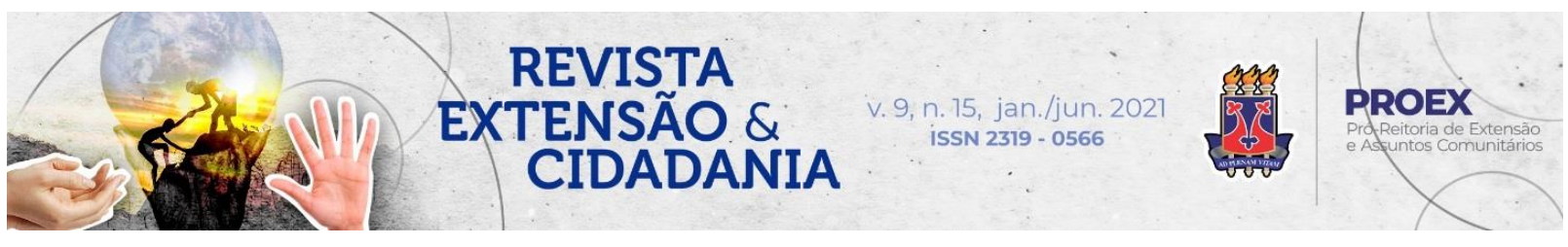

DOI: $10.22481 /$ recuesb.v9i15.8670

\title{
EDUCAÇÃO EM SAÚDE PARA INTEGRANTES DO TIRO DE GUERRA: EXPERIÊNCIA ENTRE UNIVERSIDADE E EXÉRCITO BRASILEIRO
}

\author{
Health education for members of Tiro de Guerra: experience between university \\ and brazilian army
}

\author{
César Quadros Maia ${ }^{1}$ \\ Maria Alice Guadalupe 2 \\ Ingrid Morselli Santos ${ }^{3}$ \\ Karina Aparecida Resende ${ }^{4}$ \\ Heber Paulino Pena ${ }^{5}$ \\ Maira de Castro Lima ${ }^{6}$
}

Resumo: O Exército Brasileiro é uma importante estrutura para a defesa nacional e o Tiro de Guerra é parte da constituição do serviço militar. Os atiradores devem ter formação em educação em saúde, anatomia e primeiros socorros qualificada para o desenvolvimento seguro das atividades práticas. A universidade pode colaborar com os militares na formação dos atiradores através de ações extensionistas. Esse relato de experiência tem como objetivo descrever as ações de educação em saúde realizadas por um programa de extensão da Universidade Federal de São João Del-Rei, campus CentroOeste Dona Lindu, no Tiro de Guerra de Divinópolis - Minas Gerais. As ações foram realizadas no segundo semestre de 2018, com 80 atiradores que participaram de encontros teóricos e práticos em educação em saúde com foco nos primeiros socorros. Para avaliar a satisfação dos atiradores, um questionário seguindo a escala Likert e respostas dicotômicas SIM ou NÃO, foi aplicado após os encontros. Os resultados mostraram que $98 \%$ dos atiradores concordaram que o conteúdo ministrado foi

\footnotetext{
${ }^{1}$ Graduando de Medicina, pela Universidade Federal de São João del-Rei, campus Centro-Oeste, Divinópolis, Minas Gerais, Brasil. Orcid: 0000-0003-1354-1810. E-mail: cesar_quadros@ hotmail.com

${ }^{2}$ Graduanda de Medicina, pela Universidade Federal de São João del-Rei, campus Centro-Oeste, Divinópolis, Minas Gerais, Brasil. Orcid: 0000-0002-6293-3514. E-mail: mariaaliceguadalupe@ gmail.com

${ }^{3}$ Graduanda de Medicina, pela Universidade Federal de São João del-Rei, campus Centro-Oeste, Divinópolis, Minas Gerais, Brasil. Orcid: 0000-0002-5017-6368. E-mail: ingridmorselli@ yahoo.com.br

${ }^{4}$ Farmacêutica; Mestre, pelo Programa de Pós-Graduação em Ciências Farmacêuticas da Universidade Federal de São João del-Rei, Divinópolis, Minas Gerais, Brasil. Orcid: 0000-0001-7812-7042. E-mail: karinaresendeufsj@gmail.com

${ }^{5}$ Enfermeiro; Doutor em Ciências da Saúde, Técnico do Laboratório de Habilidades da Universidade Federal de São João del-Rei, campus Centro-Oeste, Divinópolis, Minas Gerais, Brasil. Orcid: 0000-0002-9122-6827. E-mail: heberpaulino@ufsj.edu.br

${ }^{6}$ Fisioterapeuta, Doutora em Fisiologia, Professora Associada de Anatomia Humana da Universidade Federal de São João del-Rei, campus Centro-Oeste, Divinópolis, Minas Gerais, Brasil. Orcid: 0000-0002-5403-4033. E-mail: mairacastrolima@ufsj.edu.br
} 
relevante para a própria formação e $100 \%$ que foram claras as falas dos extensionistas. Foram avaliadas como excelente a didática e o material didático utilizado por $68,8 \%$ e $73,8 \%$ dos participantes, respectivamente. Sobre o encontro prático, mais de $70 \%$ opinaram como excelente a qualidade do material utilizado, a didática e o tempo disposto para a realização de cada uma das atividades. A ação extensionista promoveu impacto positivo para os discentes e para os atiradores e possibilitou abordagem multidisciplinar ativa de construção de conhecimento.

Palavras-chave: Modelos anatômicos. Primeiros Socorros. Relação Comunidade-Instituição. Militares.

Abstract: The Brazilian Army is an important structure for national defense and the Tiro de Guerra is part of the constitution of military service. Shooters must be trained in health education, anatomy and first aid, qualified for the safe development of practical activities. The university can collaborate with the military in training snipers through extension actions. This experience report aims to describe the health education actions carried out by an extension program at the Universidade Federal de São João Del-Rei, Campus Centro-Oeste Dona Lindu, in Tiro de Guerra in Divinópolis - Minas Gerais. The actions were carried out in the second semester of 2018, with 80 snipers who participated in theoretical and practical meetings in health education with a focus on first aid. To assess the snipers' satisfaction, a questionnaire using the Likert scale and dichotomous answers YES or NO, was applied at the end of the training. The results showed that $98 \%$ of the snipers agreed that the content taught was relevant to their training and $100 \%$ that the extensionists' statements were clear. The didactics and didactic material used by $68.8 \%$ and $73.8 \%$ of the participants, respectively, were evaluated as excellent. Regarding the practical meeting, more than $70 \%$ considered the quality of the material used, the didactics and the time available to carry out each activity as excellent. The extensinist action promoted a positive impact for students and the snipers and enabled an active multidisciplinary approach to knowledge construction.

Keywords: Models, Anatomic. First Aid. Community-Institutional Relations. Military Personnel.

\section{Introdução}

O Exército Brasileiro representa uma forte estrutura no país e atua em diversos ramos (MINISTÉRIO DA DEFESA, 2017). A Constituição da República Federativa do Brasil de 1988 determina que o Exército se destina à defesa da pátria, à garantia dos poderes constitucionais e, por iniciativa de qualquer desses, à defesa da lei e da ordem. Todo brasileiro do sexo masculino é obrigado ao serviço militar, o que está previsto em leis, e o prestará de acordo com a sua situação e com a sua capacidade (LEI N. 4.375 de 17 de agosto de 1964; LEI DO SERVIÇO MILITAR BRASIL, 1988).

O Tiro de Guerra (TG) é um órgão de formação de reserva que possibilita aos convocados a prática do serviço militar inicial em nível municipal e atua como complemento na formação dos jovens ao contribuir na formação do civismo e do patriotismo. Os jovens participantes recebem diversos tipos de instrução e participam de atividades específicas das forças armadas (BRASIL, 1988; MINISTÉRIO DA DEFESA, 2017).

Revista Extensão \& Cidadania, v. 9, n. 15, p. 119-130, jan./jun. 2021.

ISSN 2319-0566 DOI: 10.22481/recuesb.v9i15.8670 
Um dos principais fins do TG é o desenvolvimento de competências para a formação de cidadãos-soldados, benéficos à sociedade (ANDERSEN et al., 2016). Durante esse período de treinamento militar, eles adquirem conhecimento a ser aplicado em diversas situações desafiadoras. Esse treinamento, em tempo de paz ou de guerra, representa a possibilidade de um dano físico ou de um agravo à saúde (SMITH et al., 2000). O militar, no exercício de sua profissão, está sujeito a riscos decorrentes do manuseio de equipamentos, armamentos, munições e materiais perigosos, além da execução de técnicas de risco (ESTADO-MAIOR, 1997). Nesse contexto de risco, o conhecimento sobre doenças, anatomia humana e primeiros socorros é primordial, uma vez que esses saberes podem determinar a vida ou a morte (JÁNOSI, 2019).

O ensino de anatomia é descrito como um dos fundamentos das ciências médicas (SINGH, 2019). O conhecimento das estruturas anatômicas do corpo humano permite a compreensão de mudanças na morfologia e fisiologia dos sistemas orgânicos em casos de lesões. Além disso, o entendimento acerca dos sistemas corporais é essencial para a realização de adequado exame físico e o reconhecimento de situações ameaçadoras à sobrevivência (CHAN, 2019). Para que o atendimento de primeiros socorros seja realizado de maneira eficaz, capaz de reduzir danos e proteger a vida, é necessário que o socorrista aprenda a identificar alterações estruturas e funcionais. Como exemplo, em casos de acidente vascular encefálico agudo, o reconhecimento imediato de sinais e sintomas agiliza os primeiros socorros ao paciente e potencializa o sucesso do tratamento (SINGLETARY, 2020).

A assistência imediata dos primeiros socorros é capaz de salvar vidas (AWATSHI, 2019). Acidentes de naturezas diversas configuram um problema de saúde pública em todo o mundo, pois podem gerar graves ferimentos e até mesmo mortes. Nesse sentido, é necessário que os cidadãos saibam como prestar primeiros socorros básicos em situações de doença repentina, lesões ou acidentes, uma vez que o suporte médico nem sempre está disponível de maneira imediata (KARAKA, 2020). Estudos indicam que 1,8 a 5\% das mortes ocasionadas por trauma poderiam ser evitadas se as vítimas recebessem atendimento imediato para controle de sangramentos e liberação de vias aéreas (BAKKE, 2016).

A extensão universitária pode ser compreendida como um processo interdisciplinar que aborda aspectos acadêmicos, educativos, culturais, políticos e científicos a favor da comunidade (POLÍTICA NACIONAL DE EXTENSÃO UNIVERSITÁRIA, 2012). A ação extensionista é respaldada pela Constituição Federal de 1989, no Art.207, que determina que "As universidades gozam de autonomia didático-científica, administrativa e de gestão financeira e patrimonial, e

Revista Extensão \& Cidadania, v. 9, n. 15, p. 119-130, jan./jun. 2021. 
obedecerão ao princípio de indissociabilidade entre ensino, pesquisa e extensão.” (BRASIL, 1988).

Tendo em vista a importância do conhecimento da anatomia no contexto dos primeiros socorros, o programa de extensão "Ensino de Anatomia e Noções de Primeiros Socorros em Divinópolis-MG” da Universidade Federal de São João del-Rei, Campus Centro-Oeste Dona Lindu (UFSJ/CCO), promove esse tema na comunidade. Esta ação extensionista teve início em 2015, como projeto de extensão, tendo como público-alvo os militares do Corpo de Bombeiros Militar local e o objetivo de ensinar anatomia aos socorristas (RESENDE et al., 2017). O projeto ampliou o público-alvo ao longo das atividades realizadas e abrangeu o Serviço de Atendimento Móvel de Urgência e o Exército Brasileiro. Tornando-se um programa, foi acrescido ao tema noções de primeiros socorros e incluídas as instituições de ensino escolares. O objetivo desse relato de experiência é descrever as ações extensionistas de educação em saúde, com foco em primeiros socorros, para integrantes do Tiro de Guerra 04-019 de Divinópolis-MG.

\section{Metodologia}

O relato de experiência refere-se às atividades desenvolvidas no segundo semestre de 2018, para os integrantes do TG do batalhão de Divinópolis-MG. O treinamento dos convocados inclui a formação em primeiros socorros e o programa de extensão foi solicitado para ministrar esse conteúdo. A amostra total foi constituída por 80 atiradores, denominação recebida por jovens em serviço militar no TG com base na Lei do Serviço Militar. A participação nas aulas era obrigatória pelo treinamento militar.

Foram realizados três encontros teóricos de duas horas de duração, na unidade de treinamento do TG, no horário de 6:00 às 8:00 horas da manhã. E junto ao Corpo de Bombeiros Militar, um encontro prático de técnicas de primeiros socorros.

Os extensionistas foram capacitados através de reuniões semanais de estudo para abranger todo o conteúdo solicitado pelo TG na temática teórica e prática de primeiros socorros. Estudantes dos cursos de graduação em enfermagem, farmácia e medicina, da UFSJ/CCO, desenvolveram o tema com abordagem interdisciplinar priorizando a educação em saúde e o entendimento dos processos orgânicos e patológicos.

De acordo com a indissociabilidade ensino-pesquisa-extensão proposta pela ação extensionista, todo o conteúdo ministrado foi desenvolvido baseado em evidências científicas.

Revista Extensão \& Cidadania, v. 9, n. 15, p. 119-130, jan./jun. 2021. 
Foram utilizados protocolos e guias atualizados do Ministério da Saúde e livros didáticos e artigos científicos atuais na elaboração do material didático.

Após o último encontro, foi aplicado um questionário com perguntas fechadas, seguindo a escala Likert e respostas dicotômicas de SIM ou NÃO. Foi avaliado o grau de satisfação dos atiradores com relação à didática e à qualidade dos encontros, além da percepção pessoal sobre a relevância das atividades desenvolvidas. Essa avaliação teve como objetivo orientar a própria estratégia e didática utilizada pelo programa de extensão nas próximas ações. O questionário foi autoaplicável e garantiu o sigilo das informações prestadas.

Ao fim do período de encontros teóricos e práticos, os extensionistas participaram do processo de seleção de atiradores para o ano de 2019, aferindo sinais vitais dos candidatos, a convite dos militares do TG.

\section{Resultados}

O conteúdo teórico ministrado foi Anatomia e primeiros socorros; Técnicas de aplicação de curativos; Acidentes com animais peçonhentos e suas propriedades toxicológicas; Estancamento de hemorragias; Proteção de ferimentos; Acidentes produzidos pelo sol e calor; Asseio corporal e higiene; Doenças sexualmente transmissíveis; Doenças tropicais endêmicas do Brasil e da região; Anatomia óssea e correlação na prática clínica e, por último, Fisiopatologia dos sistemas cardiovascular, nervoso e respiratório, conteúdo da matriz curricular do ensino nacional do TG.

Todas as dúvidas relacionadas ao tema puderam ser discutidas pelos atiradores antes, durante e após os encontros. Houve uma relevante troca de saberes entre os extensionistas e a comunidade. Toda a didática foi voltada para a participação ativa do público-alvo na construção do conhecimento. A universidade não assumiu a postura de uma instituição detentora do saber que foi entregar um conteúdo pronto, mas viu-se como um instrumento capaz de aprender e ensinar.

Discussões foram fomentadas durante os encontros teóricos e os atiradores mostraram interesse pelo tema abordado. Os extensionistas conduziram o conteúdo em grupo multiprofissional para que todas as temáticas fossem exploradas de maneira diversa e com pontos de vista diferentes. O Laboratório de Anatomia Humana da UFSJ/CCO emprestou peças anatômicas sintéticas com intuito de facilitar a transmissão e entendimento do conhecimento.

Revista Extensão \& Cidadania, v. 9, n. 15, p. 119-130, jan./jun. 2021. 
Figura 1 - Encontro teórico em educação em saúde, com ênfase em primeiros socorros, no Tiro de Guerra. Divinópolis, Minas Gerais, Brasil, 2018. ( N=80)

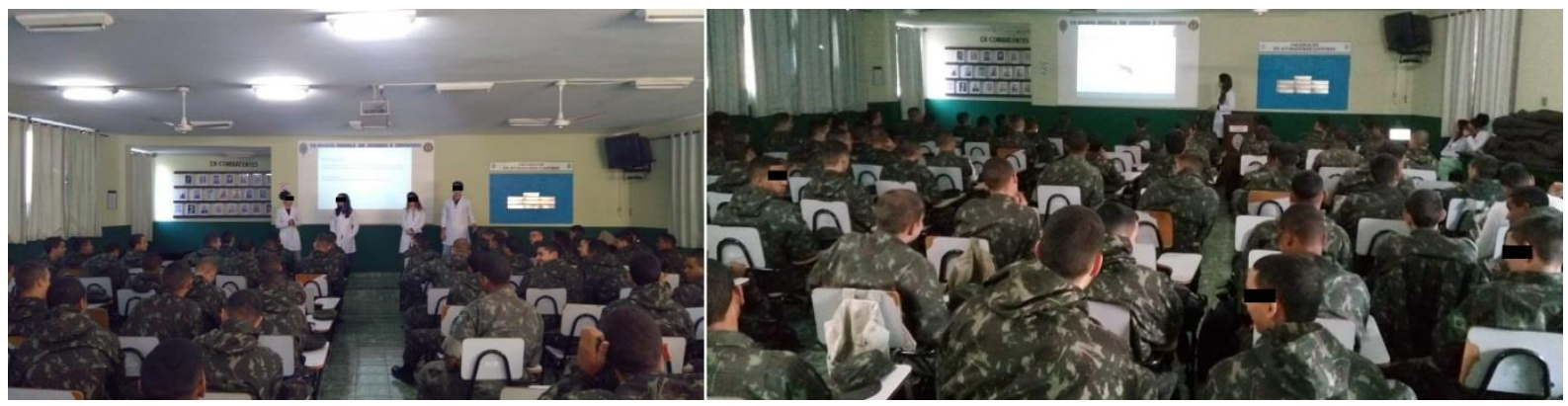

Fonte: Acervo fotográfico dos autores, 2018.

Após a experiência dos encontros teóricos, os discentes foram convidados para o encontro prático sobre primeiros socorros em situações de emergência/urgência em campo externo do TG. Essa prática foi realizada em parceria com o Corpo de Bombeiros Militar. O conteúdo abrangeu técnicas de atendimento pré-hospitalar em casos de parada cardiorrespiratória, aspiração de corpo estranho e habilidades para primeiros socorros do cotidiano. O Laboratório de Habilidades da UFSJ/CCO emprestou bonecos sintéticos para treinamento das manobras de salvamento pelos atiradores. Este material é o mesmo utilizado por estudantes dos cursos de graduação da universidade e favorece a compreensão em relação à manobra demonstrada.

Ações práticas de salvamento foram ensinadas pelos bombeiros e os extensionistas auxiliaram na realização dessa ação educativa.

Figura 2 - Encontro prático em primeiros socorros no Campo de Treinamento do Tiro de Guerra. Divinópolis, Minas Gerais, Brasil, 2018. (N=80)

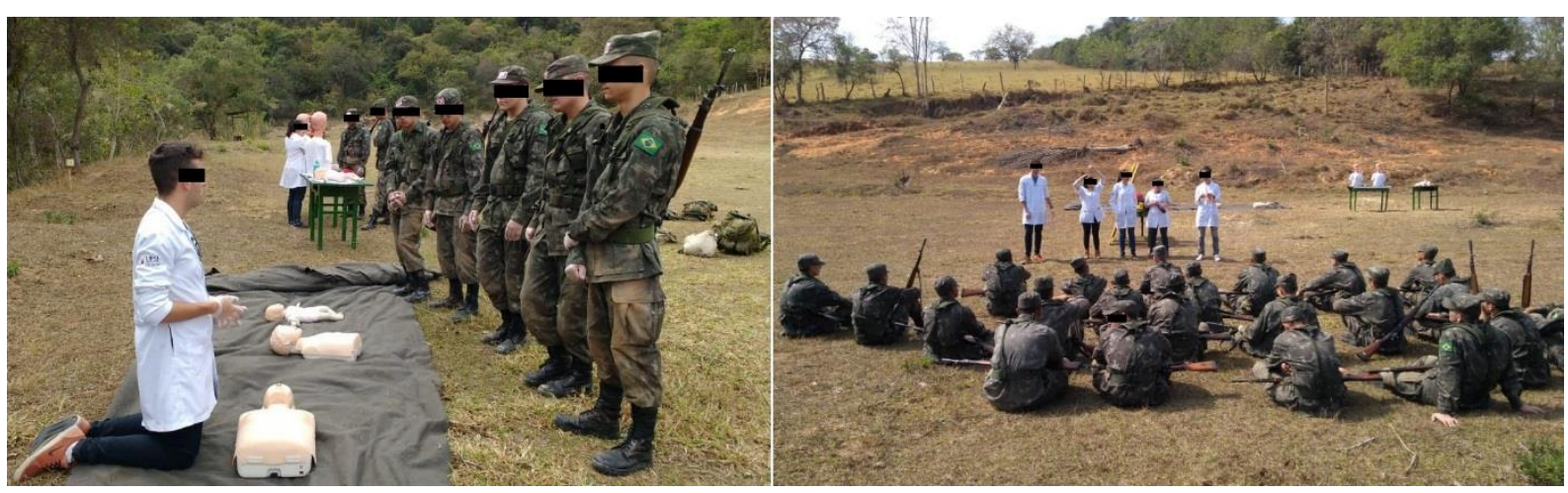

Fonte: Acervo fotográfico dos autores, 2018.

Revista Extensão \& Cidadania, v. 9, n. 15, p. 119-130, jan./jun. 2021.

ISSN 2319-0566 DOI: 10.22481/recuesb.v9i15.8670 
Posteriormente, o TG convidou o programa de extensão para auxiliar no processo seletivo dos novos atiradores para o ano de 2019. A aferição de dados vitais, como frequência cardíaca, frequência respiratória e pressão arterial foi realizada pelos extensionistas nos candidatos inscritos.

O questionário mostrou que o público-alvo ficou satisfeito com o método de ensino utilizado nos encontros teóricos. Cerca de $98 \%$ dos atiradores concordaram que o conteúdo ministrado foi relevante para a própria formação e $100 \%$ que foram objetivas as falas dos extensionistas. A maior parte das dúvidas foram respondidas antes e após as aulas, 53,8\%. A didática foi avaliada por $68,8 \%$ dos ouvintes como excelente e a mesma avaliação foi feita por 73.8\% dos participantes com relação ao material didático utilizado. Sobre as práticas de primeiros socorros, mais de $70 \%$ opinaram como excelente a qualidade do material utilizado, a didática e o tempo disposto para a realização de cada uma das atividades (Tabela 1).

\section{Tabela 1 - Frequência e porcentagem dos resultados do questionário avaliativo da ação extensionista, teórica e prática, no Tiro de Guerra do Exército. ( $\mathrm{N}=80$ )}

\begin{tabular}{|c|c|c|c|}
\hline Item do questionário & Respostas & $(\mathbf{n}=80)$ & $\%$ \\
\hline \multirow{3}{*}{$\begin{array}{l}\text { O conteúdo ministrado foi relevante } \\
\text { para sua formação? }\end{array}$} & Sim & 79 & 98,7 \\
\hline & Não & 1 & 1,3 \\
\hline & & 80 & - \\
\hline \multirow{3}{*}{$\begin{array}{l}\text { Na sua opinião foram claras as falas } \\
\text { dos professores? } \\
\text { Em relação as suas dúvidas }\end{array}$} & Sim & 80 & 100 \\
\hline & Não & - & - \\
\hline & Foram respondidas durante as aulas & 33 & 41,3 \\
\hline & Foram respondidas após as aulas & 2 & 2,5 \\
\hline \multirow{7}{*}{ Avalie quanto a didática utilizada; } & Foram respondidas antes e após as & 43 & 53,8 \\
\hline & aulas & & 2,5 \\
\hline & Não foram respondidas & 2 & \\
\hline & Ruim & 3 & 3,8 \\
\hline & Regular & 1 & 1,3 \\
\hline & Bom & 3 & 3,8 \\
\hline & Muito bom & 18 & 22,5 \\
\hline \multirow{4}{*}{$\begin{array}{l}\text { Avalie quanto a qualidade do } \\
\text { material; }\end{array}$} & Excelente & 55 & 68,8 \\
\hline & Ruim & 2 & 2,5 \\
\hline & Regular & 2 & 2,5 \\
\hline & Bom & 3 & 3,8 \\
\hline \multirow{7}{*}{$\begin{array}{l}\text { Sobre as práticas de primeiros } \\
\text { socorros avalie quanto a qualidade do } \\
\text { material; }\end{array}$} & Muito bom & 14 & 17,5 \\
\hline & Excelente & 59 & 73,8 \\
\hline & Ruim & 2 & 2,5 \\
\hline & Regular & 2 & 2,5 \\
\hline & Bom & 6 & 7,5 \\
\hline & Muito bom & 13 & 16,3 \\
\hline & Excelente & 57 & 71,3 \\
\hline
\end{tabular}

Revista Extensão \& Cidadania, v. 9, n. 15, p. 119-130, jan./jun. 2021. 


\begin{tabular}{llll} 
Sobre as práticas de primeiros & Ruim & 2 & 2,5 \\
socorros avalie ao tempo disposto & Regular & 1 & 1,3 \\
para a realização de cada uma das & Bom & 5 & 6,3 \\
atividades; & Muito bom & 13 & 16,3 \\
& Excelente & 59 & 73,8 \\
Sobre as práticas de primeiros & Ruim & 2 & 2,5 \\
socorros avalie quanto a didática & Regular & 2 & 2,5 \\
utilizada; & Bom & 3 & 3,8 \\
& Muito bom & 14 & 17,5 \\
& Excelente & 59 & 73,8 \\
\hline
\end{tabular}

Fonte: Tabela elaborada pelos autores.

\section{Discussão}

A prática extensionista é de grande importância para o crescimento pessoal e científico dos discentes, que aplicam de forma prática os conhecimentos construídos ao longo da formação acadêmica e, concomitantemente, possibilita à comunidade benefícios socioeducativos (SANTOS et al., 2016). O processo de construção do conteúdo dos temas solicitados pelo TG, que foram além da proposta do programa de extensão em anatomia e primeiros socorros, promoveu impacto na formação do estudante. Temas variados em educação em saúde foram um desafio para os universitários. Encontros semanais de estudo, discussão dos temas e construção do material didático para a matriz curricular do TG.

Nenhum estudo foi encontrado na literatura relatando contribuição da educação em saúde para integrantes das Forças Armadas, incluindo o TG. Sendo que os conhecimentos da universidade em primeiros socorros podem gerar benefícios para essas instituições de defesa do Brasil. É descrito na literatura que o conhecimento dessa temática pode salvar vidas, além de impedir a incapacidade permanente e reduzir longos períodos de hospitalização. O socorro rápido é fundamental para a equipe, pois a maioria dos militares feridos ou doentes é capaz de retornar às suas unidades quando são aplicados primeiros socorros adequados (FM, 1988).

Essa ação extensionista foi capaz de promover impacto e transformação social como foi evidenciado pela análise do questionário em que 79 atiradores concordaram que o conteúdo ministrado foi relevante para a própria formação. O conhecimento trabalhado nos encontros também pode ser difundido e aplicado a favor da sociedade através desses jovens militares. Com relação às dúvidas apresentadas pelos atiradores, os resultados mostraram que a maioria teve seus questionamentos sanados, o que evidencia o comprometimento dos extensionistas

Revista Extensão \& Cidadania, v. 9, n. 15, p. 119-130, jan./jun. 2021. 
com a qualidade do conteúdo ministrado e com a compreensão adequada do público-alvo. As respostas ao questionário mostraram que a didática utilizada foi capaz de promover o conhecimento na temática trabalhada. De acordo com Sousa (2019), a extensão deve ser capaz de contribuir para o conhecimento teórico, prático e profissional dos participantes, reforçando o caráter de democratização do saber.

A proposta de uma ação extensionista não deve ser enrijecida dentro do que a universidade julga importante para a comunidade. Pelo contrário, a sociedade deve ter voz ativa para participar ativamente da construção da ação através da interação dialógica. Além do conteúdo ministrado ter extrapolado a temática de anatomia e primeiros socorros, as ações dos extensionistas também foi além do programado.

Inicialmente, estavam previstos somente encontros teóricos, que se estenderam para a prática a convite do Corpo de Bombeiros, que são parceiros, desde o início, desse programa de extensão. Além disso, a confiança dos militares do TG no trabalho dos extensionistas resultou em uma solicitação de auxílio no processo seletivo da nova turma do TG, através da aferição de sinais vitais. Os extensionistas aprenderam na prática a responder à demanda social através do conhecimento acadêmico e dialogaram com a comunidade de maneira eficaz. A formação do discente da graduação vai além da aquisição de conhecimentos técnico-científicos, uma vez que inclui processos educativos pautados na aprendizagem ativa e na valorização da participação do indivíduo (CARABETTA, 2016).

As ações no TG promoveram práticas acadêmicas integradoras entre áreas distintas do conhecimento, os estudantes cursavam cursos diferentes e puderam debater as temáticas abordadas sob diversas perspectivas. A importância dessa troca foi mencionada por Rios, em 2019, que defende o entendimento sobre interdisciplinaridade durante a formação profissional em saúde como subsídio para a atuação em equipe. A interprofissionalidade e a interdisciplinaridade podem ser entendidas como estratégias que buscam oferecer oportunidades de aprendizado em conjunto com outras categorias profissionais (PARO, 2018; RIOS, 2019).

A indissociabilidade ensino-pesquisa-extensão deve ser trabalhada e praticada na universidade com prioridade. A ciência deve ultrapassar os muros universitários e chegar à comunidade promovendo benefícios e mudança social. Poucos são os que têm acesso direto aos conhecimentos gerados na universidade e a extensão é o meio para a democratização do acesso à ciência e a promoção do redimensionamento da função social da academia (MENDONÇA \& SILVA, 2002). O conhecimento científico em saúde pode contribuir socialmente com a criação

Revista Extensão \& Cidadania, v. 9, n. 15, p. 119-130, jan./jun. 2021. 
de políticas públicas eficientes capazes de salvar vidas. Bachmann, em 2018, defendeu a ideia que a ação extensionista é capaz de promover inovação social, compreendida como novas estratégias, como novos conceitos e como novas disposições que se adéquam às necessidades sociais de todos tipos.

\section{Considerações finais}

O trabalho articulado entre Universidade e Tiro de Guerra promoveu impacto positivo para os discentes e para os atiradores, além de possibilitar uma abordagem multidisciplinar ativa de construção de conhecimento. A extensão universitária deve ser estimulada dentro das instituições de ensino, tendo em vista que a academia deve sempre preparar o seu melhor para contribuir com a comunidade, principalmente a universidade pública que pode devolver o investimento da sociedade na forma de ações sociais relevantes. É válido apontar que as Forças Armadas estão inseridas em todo o território nacional, assim, atividades de extensão relacionadas a essas temáticas podem ser desenvolvidas em diversos municípios.

\section{Referências}

ANDERSEN, Kimberley A. et al. Musculoskeletal lower limb injury risk in army populations. Sports Medicine-Open, v. 2, n. 1, p. 1-9, 2016.

BACHMANN, Adriana Maria Reiter. Extensão universitária e inovação social: estudo em uma universidade pública municipal. Revista Gestão \& Sustentabilidade Ambiental, v. 7, n. 1, p. 447-466, 2018.

BAKKE, Håkon Kvåle et al. A nationwide survey of first aid training and encounters in Norway. BMC Emergency Medicine, v. 17, n. 1, p. 1-7, 2016.

BRASIL. Constituição (1988). Constituição da República Federativa do Brasil. Brasília, DF: Senado Federal: Centro Gráfico, 1988.

BRASIL. Lei $\mathbf{n}^{\mathbf{0}}$ 4.375, de 17 de agosto de 1964. Lei do Serviço Militar. Disponível em: http://www. planalto. gov. br/ccivil_03/leis L, v. 4375. Acesso em: 20 mar. 2021.

BRASIL. Ministério da Defesa. História do Exército. Exército Brasileiro. Departamento Geral de Educação e Cultura do Exército. 2017.

BRASIL. Ministério da Saúde. Guia de vigilância em saúde: volume único. 2017.

Revista Extensão \& Cidadania, v. 9, n. 15, p. 119-130, jan./jun. 2021.

ISSN 2319-0566 DOI: 10.22481/recuesb.v9i15.8670 
BRASIL. Ministério do Exército. Estado-Maior do Exército. Manual Técnico. Manual do instrutor T 21-250. 3. ed. 1997.

CARABETTA JR, Valter. Metodologia ativa na educação médica. Revista de Medicina, v. 95, n. 3, p. 113-121, 2016.

CHANG CHAN, Ana Yoe-Cheng et al. Approaches of anatomy teaching for seriously resource-deprived countries: a literature review. Education for Health, Abingdon, England, v. 32, n. 2, p. 62-74, 2019.

FM 21-11. First Aid for Soldiers. 1988.

FÓRUM DE PRÓ-REITORES DE EXTENSÃO DAS UNIVERSIDADES PÚBLICAS BRASILEIRAS; FÓRUM DE PRÓ-REITORES DE EXTENSÃO DAS UNIVERSIDADES PÚBLICAS BRASILEIRAS. Política nacional de extensão universitária. 2012.

JÁNOSI, András et al. Szívinfarktus miatt kezelt betegek prehospitális késési idejének elemzése. Orvosi Hetilap, v. 160, n. 1, p. 20-25, 2019.

KARACA, Ayda; KÖSE, Selmin. The effect of knowledge levels of individuals receiving basic first aid training in Turkey on the applications of first aid. Nigerian Journal of Clinical Practice, Istambul, v. 23, ed. 10, p. 1449-1455, 2020.

MENDONÇA, Sueli Guadelupe Lima; SILVA, P. S. Extensão Universitária: uma nova relação com a administração pública. Extensão Universitária: ação comunitária em universidades brasileiras. São Paulo, v. 3, p. 29-44, 2002.

PARO, César Augusto; PINHEIRO, Roseni. Interprofissionalidade na graduação em Saúde Coletiva: olhares a partir dos cenários diversificados de aprendizagem. Interface Comunicação, Saúde, Educação, v. 22, Supl. 2, p. 1577-1588, 2018.

RESENDE, Karina Aparecida et al. A Importância do Ensino de Anatomia Humana na Formação de Profissionais do Corpo de Bombeiros Militar. Revista Brasileira de Extensão Universitária, v. 8, n. 3, p. 159-165, 2017.

RIOS, David Ramos da Silva et al. Diálogos interprofissionais e interdisciplinares na prática extensionista: o caminho para a inserção do conceito ampliado de saúde na formação acadêmica. Interface - Comunicação, Saúde, Educação, v. 23, p.1-20, 2019.

SANTOS, João Henrique de Sousa et al. Extensão Universitária e Formação no Ensino Superior. Revista Brasileira de Extensão Universitária, v. 7, n. 1, p. 23-28, maio 2016.

SINGLETARY, Eunice M. et al. International consensus on first aid science with treatment recommendations. Circulation, v. 142, n. 16, suppl.1, p. 284-334, 2020.

SINGLETARY, Eunice M. et al. 2020 International consensus on first aid science with treatment recommendations. Circulation, v. 142, n. 16, suppl.1, p. 284-334, 2020.

Revista Extensão \& Cidadania, v. 9, n. 15, p. 119-130, jan./jun. 2021. 
SMITH, Gordon S. et al. Hospitalization due to injuries in the military: evaluation of current data and recommendations on their use for injury prevention. American Journal of Preventive Medicine, v. 18, n. 3, p. 41-53, 2000.

Recebido: 10.05.2021

Aceito: 19.06.2021

Revista Extensão \& Cidadania, v. 9, n. 15, p. 119-130, jan./jun. 2021. 